\title{
The relationship between periodontal status and rheumatoid arthritis - systematic review
}

\author{
Joanna Samborska-Mazur ${ }^{1}$, Dorota Sikorska ${ }^{2}$, Marzena Wyganowska-Świątkowska ${ }^{1}$ \\ ${ }^{1}$ Department of Dental Surgery and Periodontology, Poznań University of Medical Sciences, Poland \\ ${ }^{2}$ Department of Physiotherapy, Rheumatology and Rehabilitation, Poznań University of Medical Sciences, Poland
}

\begin{abstract}
There have been numerous publications investigating the relationship between periodontitis (PD) and rheumatoid arthritis (RA) so far.

This publication presents the common risk factors for the development of PD and RA. The major impact of the pathological bacterial factor and cigarette smoking with chronic inflammation playing the key role in both diseases has been confirmed by numerous studies in various populations over the years. More research focuses nowadays also on the role of improper diet and obesity. Pathophysiological pathways, such as increased concentration of proinflammatory cytokines, indirectly affecting the cardiovascular complications and coagulation disorders, which has an impact on function disorders of tissue metalloproteinase inhibitors and the plasminogen activation system, were also researched.

This systematic review of current literature has shown numerous discrepancies in previous analyses and the need for further detailed research on the relationship between periodontal status and RA.
\end{abstract}

Key words: rheumatoid arthritis, risk factors, periodontitis.

\section{Introduction}

Periodontitis (PD) is a chronic inflammation affecting the tooth-supporting tissues - gingiva, periodontal ligament, cementum on the root surface and alveolar bonecausing clinical attachment and bone loss, consequently leading to tooth loss [1]. Rheumatoid arthritis (RA) is a systemic chronic autoimmune disease. The nonspecific inflammation presents first in synovial membrane, affecting joints usually symmetrically. Progression of synovitis to systemic inflammation occurs in extra-articular manifestations and systemic complications [2].

Both diseases probably share a common etiopathogenesis and common risk factors - genetic, environmental and behavioral. It might have an impact on development and modify the progress of PD as well as RA, which was discussed in previous articles [3, 4].

Presence of chronic inflammation is crucial for progression of both conditions. Numerous studies on patients with PD and RA confirm increased levels of proinflammatory cytokines, decreased levels of tissue metalloproteinase inhibitors, increased levels of prostaglandins and an increased number of inflammatory cells, which affect chronic bone destruction [5-7].

This manuscript is a systematic review based on PubMed, in relation to the key words: periodontitis and rheumatoid arthritis, periodontal pathogens and rheumatoid arthritis, peptidylarginine deiminase and periodontitis and rheumatoid arthritis, PAS system and periodontitis and rheumatoid arthritis.

The phrases used for searching articles were developed in preliminary tests to capture the greatest number of relevant studies. The exclusion criteria were the language of the article other than Polish or English and lack of access to the full text. The selected studies were then assessed by two reviewers (dentist and rheumatologist). First, the papers were assessed with respect to the main thematic category, and general aspects. Then a detailed analysis of selected articles was made. From 190 indicated, 60 full text papers were included by the authors. 


\section{Common risk factors for periodontitis and rheumatoid arthritis}

\section{Periodontal biofilm}

Citrullination of proteins is a posttranslational conversion of peptidylarginine to peptidyl citrulline by endogenous peptidylarginine deiminases (PAD), which occurs naturally in different physiological processes, as well as in numerous pathological processes, including inflammation and autoimmunity. Chronic gingival and periodontal inflammation is associated with increased local citrullination and PAD expression in PD. From all pathogens identified to date in periodontal biofilm, there are two periopathogens - Porphyromonas gingivalis (P.g.) and Aggregatibacter actinomycetemcomitans (A.a.) - which have been suggested to be linked to anticitrullinated protein antibodies in patients with RA [8].

The "two-hit" RA model is proposed, based on the breakdown of tolerance to specific citrullinated peptides produced in gingivitis, followed by the spread of the epitope to other citrullinated host proteins into arthritis. One of the factors causing gingivitis may be the P.g., the major pathogen in chronic PD, but also isolated in biofilm samples from patients suffering from aggressive PD. Porphyromonas gingivalis has the ability to express PAD post-translational modification of arginine residues. The presence of citrulline residues in proteins such as collagen type II, fibrin, fibrinogen, vimentin and $\alpha$-enolase initiates immune responses to generate anticitrulline antibodies against these proteins [9].

In RA patients, the expression levels of peptidylarginine deiminases (PAD-2 and PAD-4) are probably correlated with the intensity of inflammation and both enzymes are demonstrable within or in the vicinity of citrullinated fibrin deposits [10].

Other virulence factors of P.g., including lipopolysaccharide (LPS), cysteine proteases (gingipains), hemagglutinins and fimbriae, lead directly to chronic inflammation, skipping of citrullination, by inducing the production of multifunctional proinflammatory cytokines: interleukin 1 and 6 (IL-1, IL-6) and tumor necrosis factor $\alpha$ (TNF- $\alpha)$, which play a key role in pathogenesis of RA and PD [11].

However, it has been suggested that even if increased citrullination and PAD-2 and PAD-4 expression in PD have been detected, they may be independent from the presence of periodontal pathogen P.g. and A.a. leukotoxins [8].

\section{Smoking}

Smoking is the major environmental risk factor for $\mathrm{RA}$, inducing the production of three autoantibodies: anti-citrullinated protein antibody (ACPA), anti-carbamylated protein antibody (ACarPA) and rheumatoid factor (RF). The appearance of these antibodies is also affected by expression of genetic factors, such as primarily the major histocompatibility complex (MHC) and polymorphism of its HLA-DRB1 locus, but also by nonMHC proteins PTPN22 and PAD-4 [12]. In patients with RA, with the combination of the environmental factor, which is smoking history, and the presence of double copies of HLA-SE genes as a genetic factor, the risk for the presence of RF increased 21-fold compared to the risk among nonsmokers carrying no SE genes [13].

Smoking has also been shown to be one of the major risk factors for periodontal disease. Studies comparing periodontal status and salivary proteolytic activity of smokers and former smokers (who did not smoke for at least 6 months) demonstrated higher salivary $\mathrm{pH}$ and better buffering capacity in non-smokers [14]. In other research, less periodontal tissue affected by the pathological condition and lower values of clinical periodontal parameters - probing pocket depth and clinical attachment loss - in non-smokers and even former smokers, compared to active smokers, has been noted. The relationship of the increased risk of periodontal disease to quantity and duration of cigarette smoking has also been demonstrated [15].

The increased prevalence of periodontal disease in patients who are smokers with serologically positive RA was investigated. The patients with ACPA antibodies clearly showed more frequent occurrence of PD among smokers compared to non-smokers, similarly to patients with RF antibodies. The risk of PD increased even further in smokers double positive for ACPA and RF antibodies, being highest among male patients [16].

However, the biological mechanisms responsible for the destructive effects of smoking on periodontal health are unclear. Tobacco smoking seems to induce changes ranging from decreased leukocyte chemotaxis to decreased production of immunoglobulins, but also to cause a stronger inflammatory reaction with increased release of potentially tissue-destructive substances such as reactive oxygen species, collagenase, serine proteases and proinflammatory cytokines. The current study indicates $\alpha 7$ nicotinic acetylcholine receptor ( $\alpha 7-n A C h R)$ as able to mediate the potential synergistic effect of nicotine and inflammation [17].

\section{Diet}

Another important environmental risk factor for both diseases is an improper diet. Eating more whole grains, fish and seafood, vegetables and fruits, reducing the consumption of red meat, sugar and highly processed products decreases the risk of occurrence of 
acute symptoms of PD and RA $[18,19]$. An improper diet may affect both diseases also indirectly - it may cause obesity, which is another risk factor for the development of RA and PD at any age [20, 21].

The iron metabolism in RA and PD patients has also been investigated. Increased serum levels of transferrin have been demonstrated in patients with PD and elevated hepcidin levels in patients with RA, which indicates a link between both diseases and iron deficiency [22, 23]

Vitamin C levels also play an important role in many diseases. Significantly lower vitamin C intake and lower blood-vitamin $C$ levels in patients with PD compared to the controls and the negative effect of low vitamin $C$ levels on the disease progression have been observed. The intervention using vitamin $\mathrm{C}$ administration after the diagnosis of periodontal disease improved gingival bleeding in gingivitis but did not exert any positive impact on PD [24].

In patients over 60 years of age suffering from RA a link between increased vitamin C intake and increased vitamin $D$ serum levels, and reduced risk of $P D$, was found [25]. The effect of vitamin D deficiency on the development of RA and PD is likely due to its key role in phosphate and calcium hemostasis and in proinflammatory cytokine inhibition controlling the correct condition of bone structures. However, many studies have excluded the association of vitamin D levels with RA and PD, and therefore its role in these diseases is not clear [26].

\section{Diabetes}

Both type 1 and type 2 diabetes are associated with increased levels of IL-6, TNF- $\alpha$ and C-reactive protein (CRP), which also occur in the pathway of RA and PD. Moreover, diabetes type 1 and RA are both autoimmune diseases and share a common genetic risk factor - the 620W allele of PTPN22 [27]. The associations of PD and RA with diabetes mellitus are two-way relationships diabetes increases the risk of occurrence and severity of PD [28]. It is also a risk factor for RA development [29].

On the other hand, PD negatively affects glycemic control - it is associated with higher glycated hemoglobin $(\mathrm{HbAlc})$ and fasting blood glucose levels, increasing the risk of diabetes development or worsening its complications [30]. Rheumatoid arthritis is also associated with increased risk of diabetes mellitus occurrence. However, the treatment of RA patients with disease-modifying anti-rheumatic drugs (DMARDs) has a positive effect on their glucose metabolism [31].

\section{Cardiovascular diseases}

Due to systemic consequences, bacteremia, increased levels of CRP and IL-6 in chronic inflammatory diseases such as RA, psoriasis, systemic lupus erythematosus or periodontal disease, the risk of developing cardiovascular abnormalities also increases. In patients with RA the risk of cardiovascular disease is 1.5-2 fold higher than in age- and gender-matched individuals from the general population [32].

Many studies of both PD and RA showed increased risk of hypertensive heart disease, ischemic heart disease, stroke, atrial fibrillation or flutter and myocardial infarction [33]. In patients with moderate and severe PD the risk of myocardial infarction was even two to four times higher [34].

\section{Stress}

Stress plays an important role in progression of chronic diseases such as RA, by release of neurotransmitters (norepinephrine) and hormones (cortisol), which indirectly affect the immune system and may increase proinflammatory cytokine levels. Among people with diagnosed post-traumatic stress disorder research has shown increased risk of developing a serologically positive form of RA [35].

The presence of proinflammatory cytokines has also been examined in former soldiers with periodontal disease suffering from post-traumatic stress disorder. There was a link between diagnosed post-traumatic stress and increased interleukin 6 level as well as Actinobacillus actinomycetemcommitans and Peptostreptococcus spp. were more frequently isolated from these patients, which indicates increased risk of PD [36].

\section{Age}

In both cases (PD and RA), one of the unmodifiable risk factors is age over 60 years. In studies on periodontal health in the USA population, sites with clinical attachment loss $\geq 3 \mathrm{~mm}$ were present in nearly $96.4 \%$ of patients over 65 years old, and $62.3 \%$ of them had one or more sites with clinical attachment loss of $\geq 5 \mathrm{~mm}$. Almost half of the older adults had at least one site with periodontal probing depth $\geq 4 \mathrm{~mm}$ and over $10 \%$ had one or more sites with periodontal probing depth $\geq 6$ $\mathrm{mm}$ [37].

In patients with RA the prevalence of anticitrullinated protein antibodies and rheumatoid factor, connected with more frequent seropositive character of the illness, increases with age. Also, more progressive bone loss has been diagnosed in people with RA over 60 years of age compared to younger people [38]. Studies of males with RA between 60 and 85 years of age also showed significantly increased odds of gingival disease, which prove the mutual influence of both diseases on each other's course [25]. 


\section{Common pathophysiological pathways for periodontitis and rheumatoid arthritis \\ Proinflammatory cytokines}

Increased levels of proinflammatory cytokines such as IL-1, IL- 6 and TNF- $\alpha$ are observed in both diseases. They have been observed in the synovial fluid of inflamed joints in patients with RA, and in the gingival fluid of patients with chronic PD [39, 40].

Interleukin 1 plays an important role in processes of initiating and sustaining an inflammatory response. It increases the production of cell adhesion molecules, facilitating the migration of leukocytes, stimulates the production of other inflammatory mediators and metalloproteinases, activates $\mathrm{T}$ - and B-lymphocytes, stimulates osteoclasts leading to bone resorption and affects cell apoptosis, limiting tissue regenerative capacity [41].

Interleukin 6, mainly produced by monocytes and macrophages, shows multidirectional action, is involved in B-cell differentiation, and together with IL-1 stimulates T-cell proliferation. IL- 6 may affect synovitis and joint damage by stimulating neutrophil migration and osteoclast maturation. IL-6 may also cause activation of polyclonal B-cells together with the production of rheumatoid factor, acute phase protein synthesis and thrombopoiesis [39].

Tumor necrosis factor alpha (TNF- $\alpha)$, secreted predominantly by monocytes and macrophages, regulates the production of collagenases, prostaglandin E2, chemokines and cytokines, cell adhesion molecules and bone resorption-related factors. Together with IL-1, it af fects bone resorption through joint activation of osteoclasts [40].

\section{Metalloproteinases}

Matrix metalloproteinases (MMPs) are a group of enzymes that are principal mediators of extracellular matrix proteins and basement membrane destruction. The proper functioning of MMPs is important in physiological processes, such as tissue remodeling, bone and tooth development, and wound healing. Especially gelatinases, including the MMP-2 and MMP-9 enzymes, play a crucial role in hemostasis and the inflammatory response. Gene polymorphisms of metalloproteinases and their expression changes are observed in both PD and RA $[42,43]$.

Metalloproteinase 9 (gelatinase B, MMP-9), induced and secreted by neutrophils, macrophages and fibroblasts, activates cytokines and chemokines in tissue remodeling. It allows the migration of secreting cells from blood vessels to the inflammation source, which significantly affects the regulation of the immune system. MMP-9 is therefore under physiological conditions one of the key enzymes in the inflammatory response. However, its elevated levels can also cause vascular fibrosis observed in cardiovascular diseases [44].

The enzyme MMP-2 plays a similar role. It is able to degrade collagen IV, non-collagen components of the cell matrix and affects fibroblast growth factor; therefore it is observed at elevated concentrations in many cardiovascular diseases [45].

Although in 2016, analysis of 17 publications showed no effect of polymorphisms of metalloproteinases on the progression of PD, numerous studies have proved their impact on periodontal tissues [46]. MMP-13 metalloproteinases in PD are responsible for the destruction of gingival tissues, and MMP-8 with MMP-9 metalloproteinases affect resorption of alveolar bone and breakdown of periodontal tissues [47].

In 2018, results of studies on the effect of smoking on MMP-2 and MMP-9 levels in patients with PD showed lower expression of MMP-2 and MMP-9 in the saliva, but increased serum concentration in smokers compared to non-smokers, which proves a different effect of nicotine on tissues [48].

Other studies among patients with PD - comparing smokers and patients with type 2 diabetes - showed increased expression of MMP-2 and MMP-9 in patients with diabetes, which is another important risk factor [49]. MMP-2 and MMP-9 levels have also been examined in postmenopausal women with diagnosed osteopenia - a link between increased gelatinase levels and bone density reduction in these women was found [50].

In RA, collagenases play a key role in the early stage of the disease: MMP-1 and MMP-13, leading to destruction of the synovium and articular cartilage, as well as MMP-2, MMP-3, MMP-8 and MMP-9 (the last two increased especially in the advanced stages of the disease), responsible for the degradation of non-collagen components of joint cells [51].

\section{Plasminogen activation system}

The plasminogen activation system (PAS) is responsible for the proper blood flow in the blood vessels, plays a key role in the early stages of wound healing and tissue remodeling (by clot formation and fibrinolysis), in inflammation, cell migration, the post-infective immune response and angiogenesis. In addition to plasminogen and plasmin, PAS includes 2 types of plasminogen activators (PA): urokinase (UPA) or its receptor (UPAR) and tissue activator (tPA); and two plasminogen activator inhibitors (PAI): PAI-1 and PAI-2. The UPA activator is the most important trigger factor for plasmin formation during cell migration and invasion, while tPA may play an important role in controlling endovascular fibrin degradation [52]. 
Plasminogen activator inhibitors 1 and 2 are responsible for the regulation of plasminogen activators (PA). In the case of overexpression of UPA and TPA, PAI-1 and $\mathrm{PAI}-2$ restrain the production of extracellular matrix (ECM), inhibiting intravascular degradation of fibrin. Plasminogen activation inhibitors are therefore responsible for hemostasis of the coagulation system. Abnormal expression of PAI-1 may be observed in numerous of pathological processes, including the course of PD and RA $[53,54]$.

Plasminogen activator inhibitors 1 and 2, affecting the migration of macrophages, are associated with the early phase of wound healing and blood clot formation in the gingival fissure, as well as with granulation tissue formation. PAI-1 stimulates differentiation of periodontal ligament stem cells into cementoblasts, which may suggest its impact in regeneration of periodontal tissues. Deficiency of plasmin or PAI-1 causes spontaneous gingival hemorrhage and destruction of tooth-supporting tissues [52].

Patients with PD have significantly increased levels of UPA and PAI-1 compared to healthy patients [52]. The concentration of the soluble receptor for urokinase plasminogen activator (sUPAR), which is associated with activation of the immune system in various diseases, was also studied. Increased suPAR levels have been observed in patients with gingivitis and PD compared to healthy people, in gingival fluid, in saliva and in serum $[55,56]$. Increased concentrations of tPA and PAI-2 have also been found in the gingival fissure fluid of patients with PD, showing the highest levels in patients suffering additionally from RA [57].

In patients with RA also increased tPA and PAI-1 levels compared to healthy people have been observed. With increasing severity of the disease, an increase in the levels of PAI-1 and a decrease in the levels of tPA were demonstrated, with the displacement of balance between them in favor of the inhibitor, which explains the fibrinolysis disorders in patients with RA [58].

Clinical studies have also shown the overexpression of UPA and its receptor UPAR in synovial fluid of patients with RA, and the protection of their antibody-mediated neutralization against the induction or progression of arthritis [59]. As in patients with PD, increased SUPAR levels were found in patients with RA, showing also increased CRP and erythrocyte sedimentation rate (ESR) values. In studies among patients in remission in RA with proper values of CRP and ESR, SUPAR values were lower than in patients with active disease but still elevated compared to healthy individuals. An increase in suPAR concentration also correlates with an increase in the DAS28 (Disease Activity Score-28) index classifying disease severity [60].

\section{Conclusions}

There are probably as many studies showing numerous differences between PD and RA as studies confirming their correlation. Common proinflammatory factors affecting the development of both diseases, bacterial, genetic and environmental factors modifying their progression, as well as many common comorbidities, lead not only to the conclusion of their common pathogenesis, but also to the possible mutual influence between PD and RA.

This review shows the multidimensionality of the connections between both diseases and reveals the need for further detailed research.

The authors declare no conflict of interest.

\section{References}

1. Könönen E, Gursoy M, Gursoy UK. Periodontitis: A Multifaceted Disease of Tooth-Supporting Tissues. J Clin Med 2019; 8: 1135, DOI: 10.3390/jcm8081135.

2. Głuszko P, Filipowicz-Sosnowska A, Tłustochowicz W. Reumatoidalne zapalenie stawów. Zalecenia postępowania diagnostyczno-terapeutycznego. Reumatologia 2016; Suppl 1: 4-11, DOI: 10.5114/reum.2016.59992.

3. Reynolds MA. Modifiable risk factors in periodontitis: at the intersection of aging and disease. Periodontol 2000 2014; 64: 7-19, DOI: 10.1111/prd.12047.

4. Klareskog L, Rönnelid J, Saevarsdottir S, et al. The importance of differences; On environment and its interactions with genes and immunity in the causation of rheumatoid arthritis. J Intern Med 2020; 287: 514-533, DOI: 10.1111/joim.13058.

5. de Molon RS, Rossa C Jr, Thurlings RM, et al. Linkage of Periodontitis and Rheumatoid Arthritis: Current Evidence and Potential Biological Interactions. Int J Mol Sci 2019; 20: 4541, DOI:10.3390/ijms20184541.

6. Tang Q, Fu H, Qin B, et al. A Possible Link Between Rheumatoid Arthritis and Periodontitis: A Systematic Review and Metaanalysis. Int J Periodontics Restorative Dent 2017, 37: 79-86, DOI: 10.11607/prd.2656.

7. Ceccarelli F, Saccucci M, Di Carlo G, et al. Periodontitis and Rheumatoid Arthritis: The Same Inflammatory Mediators? Mediators Inflamm 2019: ID 6034546, DOI: 10.1155/2019/6034546.

8. Engström M, Eriksson K, Lee L, et al. Increased Citrullination and Expression of Peptidylarginine Deiminases Independently of P. Gingivalis and A. Actinomycetemcomitans in Gingival Tissue of Patients with Periodontitis. J Transl Med 2018; 16 : 214, DOI: 10.1186/s12967-018-1588-2.

9. Wegner N, Wait R, Sroka A, et al. Peptidylarginine deiminase from Porphyromonas gingivalis citrullinates human fibrinogen and a-enolase: Implications for autoimmunity in rheumatoid arthritis. Arthritis Rheum 2010; 62: 2662-2672, DOI: 10.1002/art.27552.

10. Foulquier C, Sebbag M, Clavel C, et al. Peptidyl arginine deiminase type 2 (PAD-2) and PAD-4 but not PAD-1, PAD-3, and PAD-6 are expressed in rheumatoid arthritis synovium in 
close association with tissue inflammation. Arthritis Rheum 2007; 56: 3541-3553, DOI: 10.1002/art.22983.

11. Jia L, Han N, Du J, et al. Pathogenesis of Important Virulence Factors of Porphyromonas gingivalis via Toll-Like Receptors. Front Cell Infect Microbiol 2019; 9: 262, DOI: 10.3389/ fcimb.2019.00262.

12. Ishikawa Y, Terao C. The Impact of Cigarette Smoking on Risk of Rheumatoid Arthritis: A Narrative Review. Cells 2020; 9: 475-498, DOI:10.3390/cells9020475.

13. Klareskog L, Stolt P, Lundberg K, et al. A new model for an etiology of rheumatoid arthritis: smoking may trigger HLA-DR (shared epitope)-restricted immune reactions to autoantigens modified by citrullination. Arthritis Rheum 2006; 54: 38-46, DOI: 10.1002/art.21575.

14. Liede KE, Haukka JK, Hietanen JH, et al. The association between smoking cessation and periodontal status and salivary proteinase levels. J Periodontol 1999; 70: 1361-1368, DOI: 10.1902/jop.1999.70.11.1361.

15. Jiang Y, Zhou X, Cheng L, Li M. The Impact of Smoking on Subgingival Microflora: From Periodontal Health to Disease. Front Microbiol 2020; 11: 66-78, DOI: 10.3389/fmicb.2020.00066.

16. Eriksson K, Nise L, Alfredsson L, et al. Seropositivity combined with smoking is associated with increased prevalence of periodontitis in patients with rheumatoid arthritis. Ann Rheum Dis 2018; 77: 1236-1238, DOI: 10.1136/annrheumdis-2017-212091.

17. Zhou Z, Liu F, Wang L, et al. Inflammation has synergistic effect with nicotine in periodontitis by up-regulating the expression of $\alpha 7$ nAChR via phosphorylated GSK-3 $\beta$. J Cell Mol Med 2020; 24: 2663-2676, DOI: 10.1111/jcmm.14986.

18. Salazar CR, Laniado N, Mossavar-Rahmani Y, et al. Better-quality diet is associated with lower odds of severe periodontitis in US Hispanics/Latinos. J Clin Periodontol 2018; 45: 780-790, DOI: 10.1111/jcpe.12926.

19. Skoczyńska M, Świerkot J. The role of diet in rheumatoid arthritis. Reumatologia 2018; 56: 259-267, DOI: 10.5114/ reum.2018.77979.

20. Gulati NN, Masamatti SS, Chopra P. Association between obesity and its determinants with chronic periodontitis: A cross-sectional study. J Indian Soc Periodontol 2020; 24: 167 172, DOI: 10.4103/jisp.jisp_157 19.

21. Liu Y, Hazlewood GS, Kaplan GG, et al. Impact of Obesity on Remission and Disease Activity in Rheumatoid Arthritis: A Systematic Review and Meta-Analysis. Arthritis Care Res 2017; 69: 157-165, DOI: 10.1002/acr.22932.

22. Costa SA, Moreira ARO, Costa CPS, Carvalho Souza SF. Iron overload and periodontal status in patients with sickle cell anemia: a case series. J Clin Periodontol 2020, DOI: 10.1111/ jcpe.13284

23. Chen Y, Xu W, Yang H, et al. Serum Levels of Hepcidin in Rheumatoid Arthritis and Its Correlation with Disease Activity and Anemia: A Meta-analysis. Immunol Invest 2020; 26: 1-16, DOI: 10.1080/08820139.2020.1742731.

24. Tada A, Miura H. The Relationship between Vitamin C and Periodontal Diseases: A Systematic Review. Int J Environ Res Public Health 2020; 16: 2472, DOI:10.3390/ijerph16142472.

25. Huang LG, Chen G, Chen DY, Chen HH. Factors associated with the risk of gingival disease in patients with rheuma- toid arthritis. PLoS One 2017; 12: 1-13, DOI: 10.1371/journal. pone.0186346.

26. Beyer K, Lie SA, Kjellevold M, et al. Marine omega-3, vitamin $D$ levels, disease outcome and periodontal status in rheumatoid arthritis outpatients. Nutrition 2018; 55-56: 116-124, DOI: 10.1016/j.nut.2018.03.054.

27. Liao KP, Gunnarsson M, Källberg H, et al. Specific association of type 1 diabetes mellitus with anti-cyclic citrullinated peptide-positive rheumatoid arthritis. Arthritis Rheum 2009; 60: 653-660, DOI:10.1002/art.24362.

28. Nascimento GG, Leite FRM, Vestergaard P, et al. Does diabetes increase the risk of periodontitis? A systematic review and meta-regression analysis of longitudinal prospective studies. Acta Diabetol 2018; 55: 653-667, DOI: 10.1007/s00592-0181120-4.

29. Lu MC, Yan ST, Yin WY, et al. Risk of rheumatoid arthritis in patients with type 2 diabetes: a nationwide population-based case-control study. PLoS One 2014; 9: e101528, DOI: 10.1371/ journal.pone.0101528.

30. Preshaw PM, Bissett SM. Periodontitis and diabetes. Br Dent J 2019; 227: 577-584, DOI: 10.1038/s41415-019-0794-5.

31. Nicolau J, Lequerré T, Bacquet H, Vittecoq O. Rheumatoid arthritis, insulin resistance, and diabetes. Joint Bone Spine 2017; 84: 411-416, DOI: 10.1016/j.jbspin.2016.09.001.

32. Liao KP. Cardiovascular disease in patients with rheumatoid arthritis. Trends Cardiovasc Med 2017; 27: 136-140, DOI: 10.1016/j.tcm.2016.07.006.

33. Sanz M, Marco del Castillo A, Jepsen S, et al. Periodontitis and cardiovascular diseases: Consensus report. J Clin Periodontol 2020; 47: 268-288, DOI: 10.1111/jcpe.13189.

34. Gomes-Filho IS, Coelho JMF, Miranda SS, et al. Severe and moderate periodontitis are associated with acute myocardial infarction. J Periodontol 2020, DOI: 10.1002/JPER.19-0703.

35. Maloley PM, England BR, Sayles $\mathrm{H}$, et al. Post-traumatic stress disorder and serum cytokine and chemokine concentrations in patients with rheumatoid arthritis. Semin Arthritis Rheum 2019; 49: 229-235, DOI: 10.1016/j.semarthrit.2019.02.002.

36. Aurer A, Aurer-Kozelj J, Stavljenić-Rukavina A, et al. Inflammatory mediators in saliva of patients with rapidly progressive periodontitis during war stress induced incidence increase. Coll Antropol 1999; 23: 117-124.

37. Eke PI, Wei L, Borgnakke WS, et al. Periodontitis prevalence in adults $\geq 65$ years of age, in the USA. Periodontol 2000 2016; 72: 76-95, DOI: $10.1111 /$ prd.12145.

38. Murata K, Ito $\mathrm{H}$, Hashimoto $\mathrm{M}$, et al. Elderly onset of early rheumatoid arthritis is a risk factor for bone erosions, refractory to treatment: KURAMA cohort. Int J Rheum Dis 2019; 22: 1084-1093, DOI: 10.1111/1756-185X.13428.

39. Hirano T. Interleukin 6 in autoimmune and inflammatory diseases: a personal memoir. Proc Jpn Acad Ser B Phys Biol Sci 2010; 86: 717-730, DOI: 10.2183/pjab.86.717.

40. Noh M, Jung M, Kim S, et al. Assessment of IL 6, IL 8 and TNF $\alpha$ levels in the gingival tissue of patients with periodontitis. Exp Ther Med 2013; 6: 847-851, DOI: 10.3892/etm.2013.1222.

41. Graves DT, Cochran D, The contribution of interleukin-1 and tumor necrosis factor to periodontal tissue destruction. J Periodontol 2003; 74: 391-401, DOI: 10.1902/jop.2003.74.3.391. 
42. Hsiao YF, Yang LC, Chou YS, et al. Matrix metalloproteinase-2, -9, and tissue inhibitor of MMP-2 gene polymorphisms in Taiwanese periodontitis patients. J Dent Sci 2016; 11: 411-418, DOI: 10.1016/j.jds.2016.07.001.

43. Nemec P, Goldbergová M, Swobodnik T, et al. Polymorphism of gene promotor region for MMP-2 in rheumatoid arthritis. Vnitr Lek 2006; 52: 348-354.

44. Yabluchanskiy A, Ma Y, lyer R, et al. Matrix Metalloproteinase-9: Many Shades of Function in Cardiovascular Disease. Physiology (Bethesda) 2013; 28: 391-403, DOI: 10.1152/physiol.00029.2013.

45. Hardy E, Hardy-Sosa A, Fernandez-Patron C. MMP-2: is too low as bad as too high in the cardiovascular system? Am J Physiol Heart Circ Physiol 2018; 315: 1332-1340, DOI: 10.1152/ ajpheart.00198.2018.

46. Li W, Zhu Y, Singh P, et al. Association of Common Variants in MMPs with Periodontitis Risk. Dis Markers 2016; 2016 1545974, DOI: 10.1155/2016/1545974.

47. Cavalla F, Hernández-Ríos P, Sorsa T, et al. Matrix Metalloproteinases as Regulators of Periodontal Inflammation. Int J Mo Sci 2017; 18: 440-451, DOI: 10.3390/ijms18020440.

48. Şentürk RA, Sezgin Y, Bulut Ş, Özdemir BH. The effects of smoking on the expression of gelatinases in chronic periodontitis: a cross-sectional study. Braz Oral Res 2018; 32: 114, DOI: 10.1590/1807-3107bor-2018.vol32.0114.

49. Bastos MF, Tucci MA, de Siqueira A, et al. Diabetes may affect the expression of matrix metalloproteinases and their inhibitors more than smoking in chronic periodontitis. J Periodonto Res 2017; 52: 292-299, DOI: 10.1111/jre.12394.

50. Thompson DM, Lee HM, Stoner JA, et al. Loss of alveolar bone density in postmenopausal, osteopenic women is associated with circulating levels of gelatinases. J Periodontal Res 2019; 54: 525-532, DOI: 10.1111/jre.12656.

51. Yoshihara Y, Nakamura H, Obata K, et al. Matrix metalloproteinases and tissue inhibitors of metalloproteinases in synovial fluids from patients with rheumatoid arthritis or os teoarthritis. Ann Rheum Dis 2000; 59: 455-461, DOI: 10.1136/ ard.59.6.455.

52. Wehner C, Janjić K, Agis H. Relevance of the plasminogen activation system in physiology, pathology, and regeneration of oral tissues - From the perspective of dental specialties. Arch Oral Biol 2017; 74: 136-145, DOI: 10.1016/j.archoralbio.2016.09.014.

53. Wyganowska-Świątkowska M, Surdacka A, Skrzypczak-Jankun E, Jankun J. The plasminogen activation system in periodontal tissue (Review). Int J Mol Med 2014; 33: 763-768, DOI: 10.3892/ijmm.2014.1653.

54. Emonts $M$, Hazes $M$, Houwing-Duistermaat J, et al. Polymorphisms in genes controlling inflammation and tissue repair in rheumatoid arthritis: a case control study. BMC Med Genet 2011; 12: 36-44, DOI: 10.1186/1471-2350-12-36.

55. Taşdemir I, Erbak Yılmaz H, Narin F, Sağlam M. Assessment of saliva and gingival crevicular fluid soluble urokinase plasminogen activator receptor (suPAR), galectin-1, and TNF- $\alpha$ levels in periodontal health and disease. J Periodont Res 2020, DOI: 10.1111/jre.12748

56. Skottrup PD, Dahlén G, Baelum V, Lopez R. Soluble urokinase-type plasminogen activator receptor is associated with signs of periodontitis in adolescents. Eur J Oral Sci 2018; 126: 292-299, DOI: 10.1111/eos.12424.

57. Kurgan Ş, Önder C, Balcı N, et al. Gingival crevicular fluid tissue/blood vessel-type plasminogen activator and plasminogen activator inhibitor-2 levels in patients with rheumatoid arthritis: effects of nonsurgical periodontal therapy. J Periodont Res 2017; 52: 574-581, DOI: 10.1111/jre.12425.

58. Kopeikina LT, Kamper EF, Koutsoukos V, et al. Imbalance of tissue-type plasminogen activator (t-PA) and its specific inhibitor (PAI-1) in patients with rheumatoid arthritis associated with disease activity. Clin Rheumatol 1997; 16: 254-260, DOI: 10.1007/BF02238960.

59. Buckley BJ, Ali U, Kelso MJ, Ranson M. The Urokinase Plasminogen Activation System in Rheumatoid Arthritis: Pathophysiological Roles and Prospective Therapeutic Targets. Curr Drug Targets 2019; 20: 970-981, DOI: 10.2174/13894501206661812 04164140.

60. Vasarhelyi B, Toldi G, Balog A. The Clinical Value of Soluble Urokinase Plasminogen Activator Receptor (suPAR) Levels in Autoimmune Connective Tissue Disorders. EJIFCC 2016; 27: $122-129$ 